\title{
Tuberculosis and other causes of uveitis in Indonesia
}

${ }^{1}$ Faculty of Medicine, Department of Ophthalmology, University of Indonesia and Cipto Mangunkusumo Hospital Kirana, Jakarta, Indonesia

${ }^{2}$ Department of Clinical Immunology, Internal Medicine, Erasmus Medical Center, Rotterdam, The Netherlands

${ }^{3}$ Department of Pulmonary Diseases, Internal Medicine, Erasmus Medical Center, Rotterdam, The Netherlands

${ }^{4}$ Respirology and Critical Illness Division, Department of Internal Medicine, University of Indonesia and Cipto Mangunkusumo Hospital Kirana, Jakarta, Indonesia

${ }^{5}$ Department of Ophthalmology, Erasmus Medical Center, Rotterdam, The Netherlands

Correspondence: R La Distia Nora, Faculty of Medicine, Departmentof Ophthalmology, University of Indonesia and Cipto Mangunkusumo Hospital Kirana, Jl. Kimia no 8, Jakarta Pusat 10430, Indonesia Tel: +622131902883 ext 8318;

Fax: +62213193 4878 .

E-mail: r.ladistianora@ erasmusmc.nl or

rina.ladistia@ui.ac.id

Received: 30 March 2017 Accepted in revised form: 10 September 2017

Published online:

3 November 2017

\begin{abstract}
Purpose The purpose of this study is to assess the causes of uveitis in Indonesia and determine the importance of tuberculosis (TB) as a cause of uveitis.

Patients and methods Prospective cohort study examining 146 consecutive new human immunodeficiency virus-negative patients with active uveitis between June 2014 and May 2015. We assessed the anatomic locations and specific causes of uveitis, as well as associations with infectious and noninfectious systemic diseases. We determined the prevalence of positive QuantiFERON Tb Gold test (QFT) results in Indonesian patients with uveitis and calculated the number of patients with active systemic TB.

Results Posterior and panuveitis were the most common anatomic entities (38\% each). Infections represented the most frequent cause of uveitis $(33 \%)$; the most prevalent were toxoplasmosis (19\%) and active systemic TB (8\%). The majority of patients were QFT positive (61\%). A specific diagnosis could not be established in $45 \%$ of the patients. At first presentation to the ophthalmologist, the majority of patients $(66 \%)$ had a visual acuity of less than finger counting at $3 \mathrm{~m}$ and already exhibited various complications of uveitis. When classifying the QFT-positive patients with unexplained uveitis into a TBrelated group, the percentage of 'TBassociated' uveitis cases increased from $8-48 \%$. Highly elevated QFT levels were observed in patients with uveitis of unknown cause and no signs of active systemic TB. Conclusions In Indonesia, infectious uveitis was the most common type of uveitis and the leading causes consisted of toxoplasmosis and TB. The association observed between highly elevated QFT results and uveitis of otherwise unexplained origins indicates that a link exists between the latent TB infection and the development of uveitis.
\end{abstract}

Eye (2018) 32, 546-554; doi:10.1038/eye.2017.231; published online 3 November 2017
R La Distia Nora ${ }^{1,2}$, R Sitompul ${ }^{1}$, M Bakker ${ }^{3}$, M Susiyanti ${ }^{1}$, L Edwar ${ }^{1}$, S Sjamsoe ${ }^{1}$, G Singh ${ }^{4}$, MP van Hagen ${ }^{2}$ and A Rothova ${ }^{5}$

\section{Introduction}

Uveitis is an ocular disorder that is associated with multiple infectious and non-infectious causes and a major cause of blindness worldwide. Determining the cause of uveitis in each patient is essential for disease management. ${ }^{1}$ Tuberculosis (TB) has historically been considered a major cause of uveitis, but the importance of TB as a primary cause of uveitis has gradually decreased in the last decades, although it has recently re-emerged. ${ }^{2-4}$

TB is an infectious disease caused by Mycobacterium tuberculosis. One-third of the world's population is thought to be infected with M. tuberculosis and in 2015, 10.4 million new infections occured. ${ }^{5}$ TB is typically viewed as a poverty-related disease and immunodeficiency, including infection with human immunodeficiency virus (HIV), is an important risk factor. The typical risk factors for extrapulmonary TB including eye involvement are HIV infection, young age, female gender, and non-white race. ${ }^{6}$

Ocular involvement has prevalence between 1.4 and $18 \%$ in pulmonary TB patients. ${ }^{7,8}$ The diagnosis of ocular TB is difficult to achieve, because access to intraocular tissues is limited, and few mycobacteria invade the intraocular environment. Previously, if a patient had unexplained uveitis, evidence of active systemic TB elsewhere in the body justified treatment for ocular TB. ${ }^{9}$ In recent years, the introduction of Interferon Gamma Release Assay (IGRA) tests has made it possible to identify patients with latent TB infections. Identifying uveitis with active and/or latent TB is important because it can lead to substantial visual loss or even blindness if left untreated. ${ }^{10,11}$ Previous reports have emphasized the presence of granulomas as a pathognomonic feature of ocular TB, whereas recent publications have shown that serpiginoid choroiditis and occlusive retinal vasculitis are ocular manifestations attributable to latent TB infection. $^{12}$ 
There are no precise data regarding ocular TB and other potential causes of uveitis in Indonesia, a country with a high prevalence of all three TB categories (ie, TB, TB/HIV and multi-drug-resistant TB) and that will carry a high disease burden for the years 2016-2020 according to the World Health Organization. ${ }^{5}$ In 2016, the prevalence rate of TB was estimated at 395 per $100000 .^{5}$ In the current prospective study, we determined the anatomical location and causes of uveitis in Indonesia and evaluated the prevalence of active and latent TB infections in the uveitis population using standard screening examinations including radiologic chest imaging and concurrent tuberculin skin tests (TSTs) and QuantiFERON-Tb Gold tests (QFTs).

\section{Subjects and methods}

We performed a prospective cohort study that included all 247 consecutive new patients with uveitis, who were referred to the Infection and Immunology Division of the Ophthalmology, Department of the Medical Faculty, Universitas Indonesia/Cipto Mangunkusumo Hospital, Jakarta, Indonesia. Our study occurred from June 2014 until May 2015 and was approved by the local medical ethics committee. All included patients provided informed consent. In all, 146 of the 247 enrolled patients were included in the final analysis. We excluded patients unwilling to participate and/or patients who did not complete the screening work-up and those who did not attend follow-up examinations $(n=72)$. Additional 29 HIV-positive patients with uveitis were seen in the same timeframe; they are reported separately.

A medical history was obtained and a full ophthalmological examination, including slit-lamp biomicroscopy and indirect ophthalmoscopy, was performed for all included patients. Color photographs were obtained, and fluorescein angiography, optical coherence tomography, and visual field tests were performed according to standard clinical practices. Uveitis cases were classified and graded according to the Standardization of Uveitis Nomenclature system.

All patients were submitted to standard screening protocols for uveitis to obtain the erythrocyte sedimentation rate and red and white blood cell counts. In addition, serological testing for HIV, syphilis, and toxoplasmosis, urine analysis, and radiological chest examination were performed. TSTs (RT23 SSI-2T.U/ $0.1 \mathrm{ml}$ Statens Serum Institute, Copenhagen, Denmark) and QFTs (Cellestis Inc., Carnegie, VIC, Australia) were also performed. In all cases, the QFT was performed before the TST. Because of a temporary lack of tuberculin supply, the TST was performed in only 115/146 (79\%) of the patients. In Indonesia, a TST result with an induration larger than $10 \mathrm{~mm}$ in diameter is considered positive. ${ }^{13}$
The QFT cut-off value was $0.35 \mathrm{IU} / \mathrm{ml} .{ }^{14}$ In HIV-positive patients; however, QFT and TST tests were not systematically applied because we had very limited supply of tests and the outcomes of laboratory tests in HIV-infected patients are not reliable. In addition, some HIV-positive patients $(13 / 29,45 \%)$ were previously diagnosed with systemic TB and treated, and their QFT and TST outcomes would not be contributory.

A radiological examination, standard postero-anterior chest $X$ ray (CXR) was performed in all patients. When needed, additional imaging examinations, including apico-lordotic CXR $(n=5)$ and chest computed tomography (CT) scans $(n=16)$, were conducted. Cases involving iris and/or choroidal granulomas, subretinal abscesses, serpiginoid choroiditis, and retinal vasculitis of unknown cause $(n=16)$ underwent chest CT scanning, because this modality can be useful for diagnosing granulomatous uveitis. ${ }^{15}$ Human leukocyte antigen B27 testing was not available at our institution at the time of this study.

Microbiological examinations (ie, sputum culture $(n=1)$, Ziehl Nielsen staining of sputum $(n=21)$, and PCR for $M$. tuberculosis in sputum $(N=10))$ were performed in selected cases. Additional PCRs of intraocular fluids was performed in 44 patients with a high suspicion of infectious uveitis etiology and no response to standard treatments with corticosteroids including herpes simplex virus $(n=10)$, varicella zoster virus $(n=3)$, cytomegalovirus (CMV, $n=18)$, Toxoplasma $(n=5)$, and M. tuberculosis $(n=37)$. PCR for M. tuberculosis was performed on samples isolated using a QIAamp DNA and Blood Mini Kit (QIAGEN GmbH, Hilden, Germany) and primers specific for IS 1660 in a thermal cycler. The products were further analyzed using gel electrophoresis.

Diagnoses of pulmonary TB were based on the Indonesian Society of Respirology (ISR)TB guidelines (see the algorithm illustrated in Supplementary Figure 1). ${ }^{16,17}$ Briefly, diagnoses of active pulmonary TB were based on a general clinical examination and microbiological and/or radiological findings. A review of chest X-ray and CT scan data was performed by two pulmonologists who are specialists in TB (one from Indonesia and one from the Netherlands), and cases were classified as active TB, prior but inactive TB and abnormalities other than TB.

Clinical diagnoses, such as Behcet's disease, Vogt Koyanagi Harada (VKH) syndrome, and Fuchs uveitis syndrome (FUS), were diagnosed according to current diagnostic criteria. All diagnoses of sarcoidosis were achieved according to current diagnostic criteria for patients with negative TB tests. Patients showing no evidence of active systemic TB but who had Mtb-PCRpositive intraocular fluids were not classified as having active systemic TB and were separately indicated.

Diagnoses of ocular toxoplasmosis were based on clinical 
manifestations including focal active chorioretinitis located adjacent to a chorioretinal scar in combination with positive serological results and a good response to antiparasitic drugs. We registered all demographic data and the results of all diagnostic and ophthalmological examinations.

All statistical analyses were performed using chisquared tests and one-way ANOVA tests. Statistical significance was defined as a $P$-value $<0.05$. We performed Bonferroni post-hoc analyses and all $P$-values shown were adjusted. Multiple regression analysis was used to compare all clinical and laboratory characteristics related to QFT values. For all calculations with visual acuity data, we used the logarithm of the minimum angle of resolution $(\log$ MAR). In bilateral cases, we used the average $\log$ MAR visual acuity; in unilateral cases, the visual acuity of the affected eye was used.

\section{Results}

The general characteristics of the included patients, causes of $\mathrm{TB}$, and its associations with systemic diseases are presented in Tables 1 and 2. The mean age at onset of uveitis was 41 years old and no difference was observed between genders. At first clinical presentation, a duration of uveitis of longer than 1 year was observed in approximately $25 \%$ of patients, and the majority of patients $(97 / 146,66 \%)$ had a visual acuity (VA) of less than finger counting at $3 \mathrm{~m}$. The majority of cases in our series were unilateral $(100 / 146,68 \%)$ and anterior uveitis was observed in 29/146 (20\%) of patients. Posterior and pan-uveitis were the most common presentations $(38 \%$ for each, Table 2).

Ocular infections were diagnosed in 48/146 (33\%) of the patients. Toxoplasmosis was the most common $(30 / 48,63 \%)$, followed by active TB-associated uveitis $(12 / 48,25 \%)$. Toxoplasmosis was the major cause of posterior uveitis $(27 / 56,48 \%)$. An association with a noninfectious systemic disease was observed in 11/146 (7.5\%) cases. The most frequently observed presentations were Behcet's disease and VKH syndrome (4/146, 3\% each). Ocular clinical syndromes were diagnosed in 21/146 (14\%) patients, among which masquerade syndromes $(8 / 146,5.5 \%)$ and FUS (4/146, 3\%) were the most common. A specific diagnosis was not established in 66/146 (45\%) cases.

Using ISR TB guidelines, active pulmonary TB was diagnosed in 12/146 (8\%) patients and evidence of previous pulmonary TB was observed in 23/146 (16\%; Table 3) patients. Patients with active pulmonary TBassociated uveitis showed no differences compared with the other groups in gender, laterality, anatomical location of uveitis, age of onset, VA at first visit, duration of uveitis or complications. A borderline association was found for

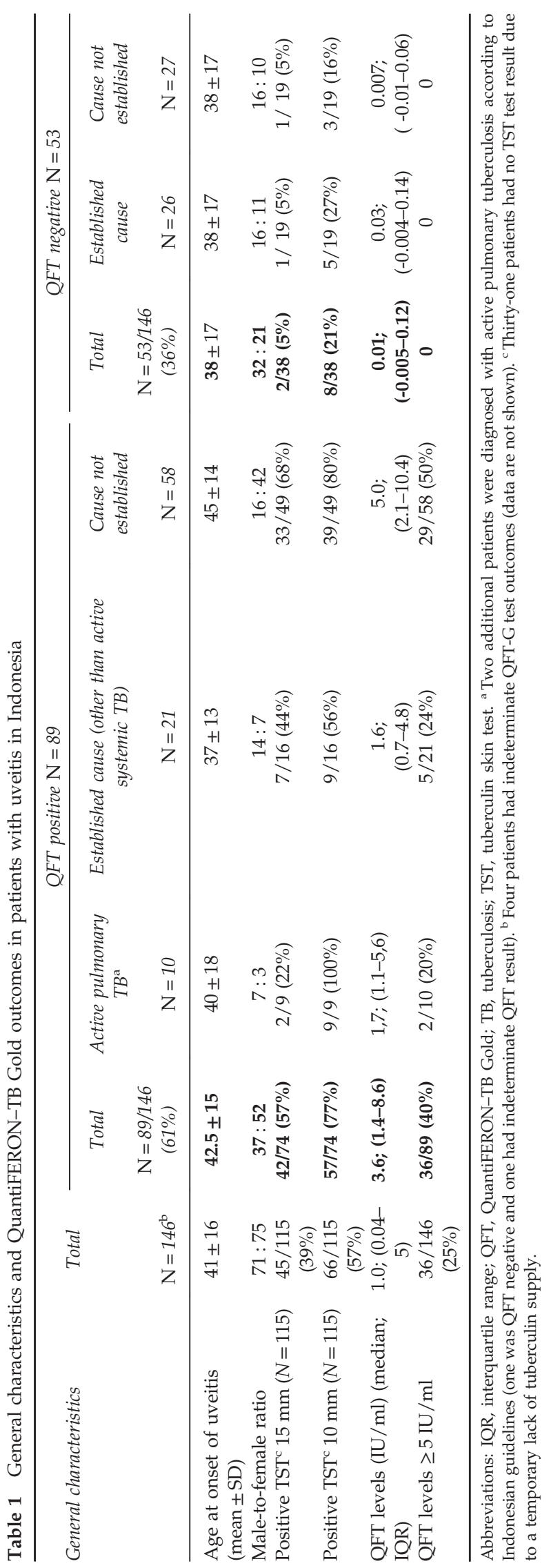




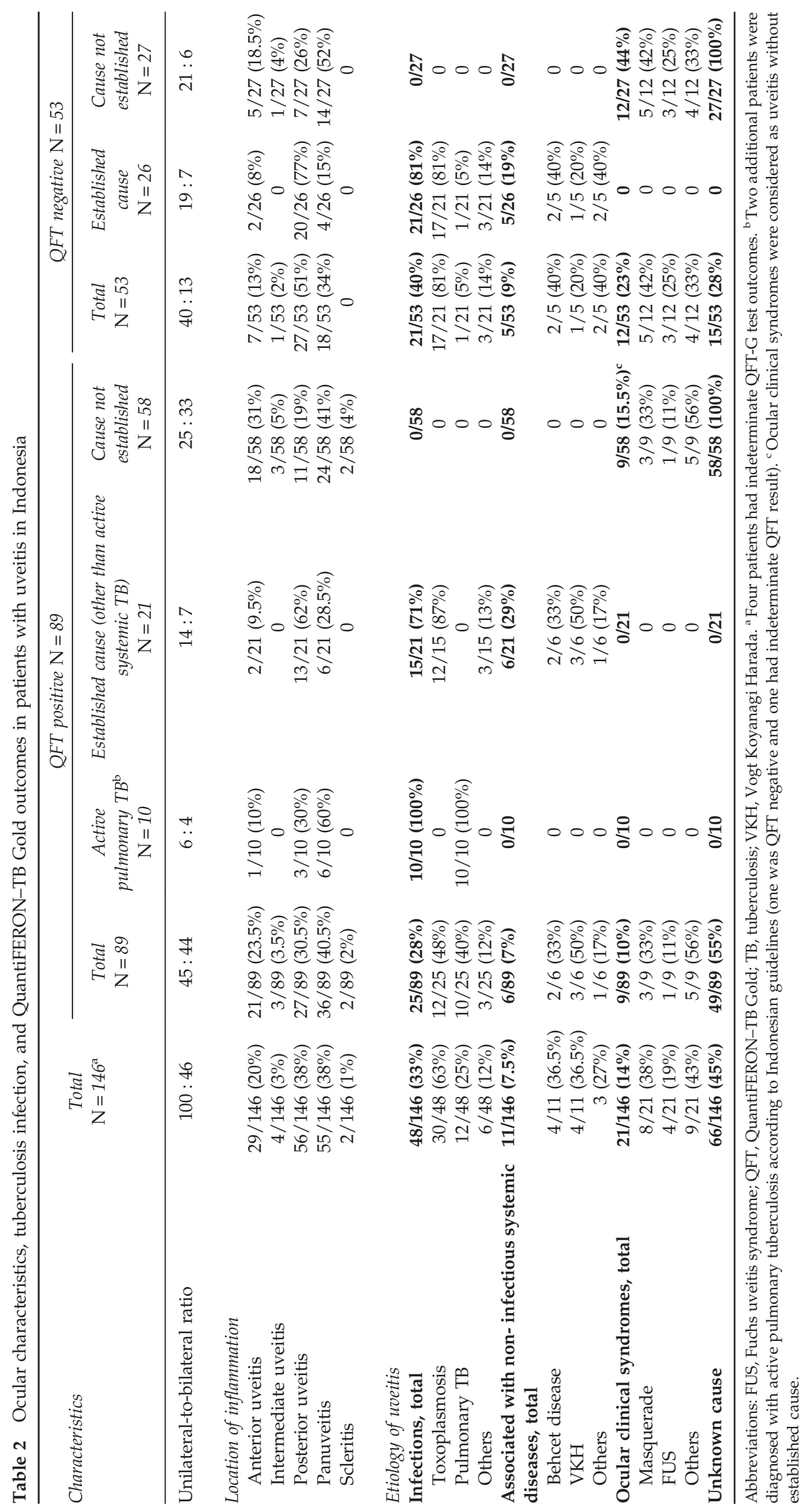


Table 3 Pulmonary tuberculosis in uveitis patients according to the Indonesian Society of Respirology Tuberculosis guidelines

\begin{tabular}{lcccc}
\hline Classification & $\begin{array}{c}\text { Total }^{\mathrm{a}} \\
\mathrm{N}=146\end{array}$ & $\begin{array}{c}\text { Active pulmonary tuberculosis } \\
\mathrm{N}=12 / 146(8 \%)\end{array}$ & $\begin{array}{c}\text { Previous pulmonary tuberculosis } \\
\mathrm{N}=23 / 146(16 \%)\end{array}$ & $\begin{array}{c}\text { No pulmonary tuberculosis } \\
\mathrm{N}=101 / 146(69 \%)\end{array}$ \\
\hline QFT-positive patients & $89 / 146(61 \%)$ & $10 / 12(83 \%)$ & $18 / 23(78 \%)$ & $54 / 101(53.5 \%)$ \\
QFT (median; IQR) & $1 ; 0.05-5.3$ & $1.5 ; 1-4.7$ & $6.2 ; 1.2-11$ & $0.5 ; 0.01-3.6$ \\
QFT $\geq 5$ IU & $36 / 146(25 \%)$ & $2 / 12(17 \%)$ & $11 / 23(48 \%)$ & $22 / 101(69 \%)$ \\
TST $\geq 15 \mathrm{~mm}$ & $45 / 115(39 \%)$ & $2 / 10(20 \%)$ & $16 / 21(76 \%)$ & $26 / 78(33 \%)$ \\
TST $\geq 10 \mathrm{~mm}$ & $66 / 115(57 \%)$ & $10 / 10(100 \%)$ & $18 / 21(86 \%)$ & $35 / 78(46 \%)$ \\
\hline
\end{tabular}

Abbreviations: IQR, interquartile range; QFT, QuantiFERON-TB Gold; TST, tuberculin skin test. ${ }^{a}$ Ten patients who could not be correctly classified according to Indonesian guidelines are not shown. ${ }^{b}$ Four patients with indeterminate QFT results were not included in the median QFT calculation.

the development of hypotonic and/or phthisical eye $\left(P=0.055, \chi^{2}\right.$-test). No specific clinical manifestations were prevalent in patients with active pulmonary TBassociated uveitis, except for choroidal granulomas $(2 / 12$, $17 \%$ vs $0 / 134,0 \%$ in other groups, $P<0.000)$. Of the two patients with serpiginoid choroiditis, one had active systemic TB and the other had previous pulmonary TB.

The majority of uveitis patients were QFT positive (89/146, 61\%; Table 2). The prevalence of QFT-positive tests was highest for the patients with active pulmonary TB-associated uveitis $(10 / 12,83 \%)$ and previous TB $(18 / 23,78 \%)$. One $(1 / 12,8 \%)$ patient with active pulmonary TB, who was diagnosed using ISR TB guidelines had a negative QFT test. The QFT was more frequently positive in female patients $(52 / 89,58 \%$ vs $21 / 53,40 \%$ in males; $P=0.038$ ), but there were no differences in age, laterality, VA at first visit, duration, clinical manifestations or complication rates of uveitis. Although unilateral cases were more prevalent in this series, QFT-positive patients were more likely to exhibit bilateral involvement than QFT-negative patients $(44 / 89$, $50 \%$ vs $13 / 53,25 \%$; $P=0.005$, multivariate binary logistic regression analysis; odds ratio, 3 ).

The QFT was more frequently positive in patients with uveitis of unknown cause than in those for whom there was an established cause other than active pulmonary TB ( $58 / 87,67 \%$ vs $21 / 47,45 \%$ in patients with an established cause; $P=0.054$, multivariate binary logistic regression analysis; odds ratio, 2). When patients with a positive QFT result and otherwise unexplained uveitis were classified as TB-related, the percentage of 'TB-associated' patients increased from $8 \%$ to $48 \%(70 / 146)(66 \%$ or $70 / 106$ of patients with infectious uveitis). Focal chorioretinal scars were more frequently observed in QFT-negative patients (22/53, $41.5 \%$ vs $17 / 89,19 \%$, $P=0.01$; odds ratio, 2.7). The specific QFT level observed in each group is indicated in Table 3. In QFT-positive patients, the QFT levels in patients with active pulmonary TB and those without pulmonary TB did not differ (median value, $1.5 \mathrm{IU}$ vs $0.5 \mathrm{IU}, P=0.570$, Mann-Whitney test). The median QFT value was higher in patients with uveitis of unknown cause than in those with an established cause (2.2 IU vs $0.3 \mathrm{IU}, P=0.008$ ). Moreover, QFT values higher than $5 \mathrm{IU} / \mathrm{ml}$ were more frequently observed in patients in which uveitis had an unknown cause than in patients with an established cause (29/85; $34 \%$ vs $5 / 46 ; 11 \%, P=0.019$; odds ratio, 3.5 ).

The TST result was $\geq 10 \mathrm{~mm}$ in $65 / 112(58 \%)$ and $\geq 15 \mathrm{~mm}$ in $44 / 112(40 \%)$ of the tested patients. The prevalence of a TST result of $\geq 15 \mathrm{~mm}$ was highest in patients with previous TB $(16 / 20,80 \%)$, while in active pulmonary TB-associated uveitis, only $2 / 10(20 \%)$ had a TST result of $\geq 15 \mathrm{~mm}(P=0.000)$. There was no association between TST positivity and gender, laterality, location of uveitis, ocular complications or clinical characteristics (except for focal chorioretinal scars, which were more commonly found in TST-negative patients: $23 / 68$ or $34 \%$ of patients had negative TST results vs $5 / 44$ or $11 \%$ of patients with positive TST results; $P=0.008$ ). All patients with active pulmonary TB had positive TST results $(\geq 10 \mathrm{~mm})$. In accordance with the QFT results, a TST value of $\geq 15 \mathrm{~mm}$ was more frequently observed in patients with uveitis of unknown origin than in those with an established diagnosis $(34 / 68,50 \%$ vs $8 / 34,23.5 \%$; $P=0.011$ ).

The QFT and TST results were highly correlated $(P<0.000)$ and this relationship was even stronger in patients with QFT levels $\geq 5 \mathrm{IU} / \mathrm{ml}$ (TST $\geq 15 \mathrm{~mm}$, $P=0.000$; TST $\geq 10 \mathrm{~mm}, P=0.001)$. Out of the 74 QFTpositive patients, $42 / 74(57 \%)$ had a TST result $\geq 15 \mathrm{~mm}$, and $57 / 74(77 \%)$ had a TST result $\geq 10 \mathrm{~mm}$.

Out of 37 PCR runs to assess the presence of $M$. tuberculosis in intraocular fluid, 4 yielded a positive result. These four patients were all QFT positive and one was classified as having active pulmonary TB (positive sputum for M. tuberculosis determined by PCR). Two patients had anterior uveitis and two had pan-uveitis. Small nodules were observed on the iris in two of these patients, but no other similarities in clinical manifestations were observed.

The characteristics of 29 HIV-positive uveitis patients are provided in Table 4 . The majority of the HIVassociated uveitis cases was of infectious origin (18/29, $62 \%)$, with CMV retinitis being most common $(9 / 18,50 \%)$ 
Table 4 Characteristics in HIV-positive patients with uveitis

\begin{tabular}{lc}
\hline Characteristic & HIV-positive patietns \\
& $\mathrm{N}=29$ \\
\hline Age (mean \pm SD), years & $37 \pm 9$ \\
Male-to-female ratio & $24: 5$ \\
Unilateral-to-bilateral uveitis ratio & $14: 15$ \\
Tuberculin skin test $>10 \mathrm{~mm}$ & $1 / 7(14 \%)$ \\
Positive QuantiFERON-TB Gold test & $1 / 2(50 \%)$ \\
& \\
Location of inflammation & \\
Anterior uveitis & $3 / 29(10 \%)$ \\
Intermediate uveitis & $2 / 29(7 \%)$ \\
Posterior uveitis & $12 / 29(41.5 \%)$ \\
Panuveitis & $12 / 29(41.5 \%)$ \\
& \\
Aetiology of uveitis & \\
Infections, total & $\mathbf{1 8 / 2 9}(\mathbf{6 2} \%)$ \\
Toxoplasmosis & $4 / 18(22 \%)$ \\
Tuberculosis & $3 / 18(17 \%)$ \\
Cytomegalovirus & $9 / 18(50 \%)$ \\
Syphilis & $2 / 18(11 \%)$ \\
Associated with non-infectious systemic & $\mathbf{0 / 2 9} \mathbf{( 0 \% )}$ \\
diseases, total & \\
Ocular clinical syndromes, total & $\mathbf{0 / 2 9} \mathbf{( 0 \% )}$ \\
Undetermined & $\mathbf{1 1 / 2 9} \mathbf{( 3 8 \% )}$ \\
\hline
\end{tabular}

Abbreviations: FUS, Fuchs uveitis syndrome; HIV, human immunodeficiency virus; QFT, QuantiFERON-TB Gold; TB, tuberculosis; TST, tuberculin skin test; VKH, Vogt Koyanagi Harada.

followed by toxoplasmosis (4/18, 22\%). Association of active systemic TB infection with uveitis at the time of presentation was found in 3/29 (10\%) cases.

\section{Discussion}

This prospective study demonstrates that the known etiology of uveitis is most commonly infectious in Indonesia. Furthermore, we show that the majority of patients consulted an ophthalmologist when uveitis had entered the late stage and they already had poor VA and complications. This late presentation limits the visual prognosis and precludes the recognition of typical clinical manifestations, which impairs the correct diagnosis of uveitis. We demonstrate that active pulmonary TB remains a frequent cause of uveitis in Indonesia (8\% of all uveitis cases) and ranks second after toxoplasmosis among infectious causes of uveitis. We also found that a majority of uveitis patients were QFT positive (61\%) and observed an excess of highly positive QFT results in patients with uveitis of unknown cause and without any signs and symptoms of active systemic TB. This finding suggests that QFT-positive uveitis might be associated with previous or latent TB, as has been previously suggested.7,18,19 Interestingly, QFT levels were much higher in patients with uveitis associated with latent and previous TB than in patients with active systemic TB- associated uveitis. The majority of patients with uveitis had a positive QFT result, illustrating the limited diagnostic value of this test in endemic populations such as ours.

In western countries, infections account for $\sim 16 \%$ of all uveitis cases. This percentage is higher in developing countries (range $20-30 \%$ ). ${ }^{3,20}$ As only proven infections are classified as infectious cases, it is likely to be that in reality these percentages are much higher. In our series, infectious causes accounted for $33 \%$ of all cases. The majority were caused by toxoplasmosis, followed by active systemic TB-associated uveitis. Our findings are similar to those described in reports from South America, Thailand, and Nepal. In contrast, reports have shown that in other countries in Asia (including India, Singapore, and Myanmar), uveitis is more often TB-associated than caused by toxoplasmosis. ${ }^{21-23}$ However, these reports classified patients with uveitis and positive QFT and/or TST results (without additional evidence of systemic TB) as having TB. Although this may explain the higher prevalence of TB-associated cases, it also prevents comparisons with our series. If the QFT-positive and unexplained cases of uveitis in our series were all classified as TB associated, the percentage of 'TBassociated' cases of uveitis would increase from 8 to $48 \%$, which illustrates the importance of classification methods. Similar disparities in the prevalence of (presumed) ocular TB can also be found in studies performed in western and middle-eastern countries (range from under 1 to $18 \%) .3,24,25$ These large variations might be caused by genuine geographical differences or potentially by a lack of appropriate classification criteria for TB-associated uveitis.

It is currently unknown whether uveitis associated with a positive IGRA test in the absence of signs of active TB represents a real ocular infection with $M$. tuberculosis, an immune reaction, or a combination of both. The presence of $M$. tuberculosis in ocular tissues has occasionally been demonstrated in active systemic TB-associated uveitis, but a histopathological study demonstrated that very few bacilli are required to cause uveitis. ${ }^{26,27}$ In India, patients with uveitis and positive IGRA outcomes had positive PCR results in the absence of systemic signs of TB infection. ${ }^{28}$ These findings show that a low-grade intraocular infection with $M$. tuberculosis is possible even in patients showing no other systemic signs of TB. The diagnostic role of performing PCR on intraocular fluids to identify TB is not yet clear and the results vary according to the selected gene targets. ${ }^{12,18,29}$ As patients can have negative PCR findings in ocular fluid while showing uveitis and active systemic TB or positive PCR findings, although showing no evidence of active systemic $\mathrm{TB},{ }^{29-32}$ dividing patients into those with either active or latent TB 
infection is inadequate. These results indicate that there is a broad spectrum of disease severity.

Leprosy is a mycobacterial disease that is endemic in Indonesia and the possibility of cross-reactivity in the QFT must be taken into account. The peptides ESAT6 and CFP10, which are included in the QFT, were reported to show positive results after infection $M$. leprae. ${ }^{33,34}$ One study conducted in the United States, a non-endemic country, reported that $5 / 10(50 \%)$ of patients with tuberculoid leprosy patients had positive QFT results, whereas none with lepromatous leprosy $(n=40)$ were QFT positive. ${ }^{35}$ As the leprosy is still prevalent in Indonesia (yearly incidence in 2015 was 7 leprosy cases per 100000 population versus 395 TB cases per 100000 population), we cannot exclude that some QFT positivity might be caused by infection with M. leprae. 5,36 However, isolated uveitis without any systemic signs of leprosy is exceedingly rare. In addition, our study included only one patient with a history of treated leprosy, and the patient's QFT result was negative.

In our series, patients with uveitis and signs of previous pulmonary TB had very high QFT levels, as did QFTpositive patients with idiopathic uveitis but no signs of systemic TB. These results are consistent with those described in previous reports. ${ }^{37-39}$ High QFT levels might reflect the induction of additional immune reactions by M. tuberculosis antigens in (genetically) susceptible patients. ${ }^{38}$ The link between uveitis and a positive IGRA test is not entirely clear and the specific pathogenesis of uveitis accompanied by a positive IGRA test remains unknown. In this study, the lack of associations between ocular features and manifestations of TB may in part be have resulted from the large number patients with endstage uveitis; it was not feasible to recognize specific clinical features in these patients.

Although this study had a prospective design, it has several limitations. First, our center provides tertiary care and it is possible that our population of patients was biased toward more severe and chronic cases. The prevalence of patients with anterior uveitis (20\%) was however very similar to that in other series performed at tertiary centers worldwide. In Indonesia, peripheral ophthalmologists commonly lack the appropriate methods to diagnose patients with uveitis and the socioeconomic status of patients frequently precludes a timely medical consultation. For example, most of the patients in our study presented with complications and already had very limited VA. This creates a complex and challenging situation for organizations in the ophthalmology health system in Indonesia. Ophthalmologists in Indonesia should be aware of the high prevalence TB- and other infection-associated uveitis. Close collaboration between infectious diseases specialists and pulmonologists is needed to distinguish between infectious and non-infectious uveitis, and to thereby enable the selection of an appropriate treatment. In addition, we did not evaluate the QFT value in HIVpositive patients due funding limitations and the unreliability of these tests in the HIV-infected population. Indeed, QFT in HIV-positive patients is reported to have low sensitivity and to frequently exhibit indeterminate results. ${ }^{40}$ Therefore, the prevalence of positive QFT outcomes in HIV-positive patients could not be compared with HIV-negative patients; nonetheless, the proportion of uveitis associated with active TB infection was similar for HIV-positive and HIV-negative patients with uveitis (3/29, $10 \%$ vs $12 / 146,8 \%$, respectively).

In conclusion, infections are the leading cause of uveitis in Indonesia and most patients consult with an ophthalmologist when their VA is already severely compromised and their clinical complications are apparent. Infectious uveitis is potentially treatable and blindness is therefore preventable in these patients. Our findings will hopefully increase the early recognition of uveitis etiologies and have a role in preventing unnecessary blindness.

\section{Summary}

What was known before

- Active ocular TB has historically been an important cause of uveitis. Recently, in western countries, active ocular TB has been found to primarily occur in immunosuppressed patients.

- The introduction of Interferon Gamma Release Assay/ Quantiferon-G tests made it possible to identify patients with latent TB infections and it seems probable that some types of uveitis are associated with latent TB infection.

- Data related to the causes of uveitis in Indonesia, a country in which TB is endemic, are entirely lacking.

\section{What this study adds}

- Infectious uveitis was identified as the most common type of uveitis in Indonesia (33\% of all cases). The leading causes were toxoplasmosis and uveitis associated with active pulmonary TB.

- Latent TB was present in $40 \%$ of patients with uveitis of unknown origin and when we classified cases of uveitis of unknown cause and latent TB as TB-associated uveitis the percentage of TB-associated uveitis increased from 8 to $48 \%$ of all uveitis cases.

- Extremely high QFT levels were found in patients with uveitis of otherwise unexplained origin, indicating a clear link between uveitis and latent TB. These data suggest the involvement of an associated (auto)immune reaction to TB antigens.

- The proportion of cases associated with active systemic TB was similar in HIV-positive and HIV-negative patients with uveitis. 


\section{Conflict of interest}

The authors declare no conflict of interest.

\section{Acknowledgements}

This project was supported by the Directorate General of Higher Education, Indonesian Ministry of Research Technology and Higher Education. We thank Yulia Aziza, Kiki Nauli, Azalia Latuasan, Dina Lestari, and Ikhwanuliman Putera for their help in collecting the data of the patients. The first author was supported by the Directorate General of Higher Education, Indonesian Ministry of Research Technology, and Higher Education during her PhD studies in Erasmus MC, The Netherlands.

\section{Author contributions}

Contributions of authors: design (AR and RLDN) and conduct the of the study (AR, RLDN, MB, and G.S.); collection (RLDN, MS, LE, SS, and GS), management (RLDN and RS), analysis (RLDN and AR), and interpretation of the data (RLDN, $\mathrm{AR}, \mathrm{MB}$, and GS); preparation (RLDN and AR), review (RLDN, AR, MB, $\mathrm{MPvH}, \mathrm{RS}, \mathrm{MS}, \mathrm{LE}, \mathrm{SS}$, and GS), and approval of the manuscript (RLDN, AR, MB, MPvH, RS, MS, LE, SS, and GS).

\section{References}

1 Suttorp-Schulten MS, Rothova A. The possible impact of uveitis in blindness: a literature survey. Br J Ophthalmol 1996; 80(9): 844-848.

2 Das D, Bhattacharjee H, Das K, Tahiliani PS, Bhattacharyya P, Bharali G et al. The changing patterns of uveitis in a tertiary institute of Northeast India. Indian J Ophthalmol 2015; 63(9): 735-737.

3 Jones NP. The Manchester Uveitis Clinic: the first 3000 patients-epidemiology and casemix. Ocul Immunol Inflamm 2015; 23(2): 118-126.

4 Luca C, Raffaella A, Sylvia M, Valentina M, Fabiana V, Marco $C$ et al. Changes in patterns of uveitis at a Tertiary Referral Center in Northern Italy: analysis of 990 consecutive cases. Int Ophthalmol 2017; e-pub ahead of print 9 January 2017; doi:10.1007/s10792-016-0434-x.

5 World Health Organization. Global Tuberculosis Report. World Health Organisation: Geneva, Switzerland, 2016.

6 Yang Z, Kong Y, Wilson F, Foxman B, Fowler AH, Marrs CF et al. Identification of risk factors for extrapulmonary tuberculosis. Clin Infect Dis 2004; 38(2): 199-205.

7 Donahue HC. Ophthalmologic experience in a tuberculosis sanatorium. Am J Ophthalmol 1967; 64(4): 742-748.

8 Bouza E, Merino P, Munoz P, Sanchez-Carrillo C, Yanez J, Cortes C. Ocular tuberculosis. A prospective study in a general hospital. Medicine (Baltimore) 1997; 76(1): 53-61.

9 Helm CJ, Holland GN. Ocular tuberculosis. Surv Ophthalmol 1993; 38(3): 229-256.

10 Bansal R, Gupta A, Gupta V, Dogra MR, Bambery P, Arora SK. Role of anti-tubercular therapy in uveitis with latent/manifest tuberculosis. Am J Ophthalmol 2008; 146(5): 772-779.e772.

11 Ang M, Hedayatfar A, Wong W, Chee SP. Duration of antitubercular therapy in uveitis associated with latent tuberculosis: a case-control study. Br J Ophthalmol 2012; 96(3): 332-336.

12 La Distia Nora R, van Velthoven ME, Ten Dam-van Loon $\mathrm{NH}$, Misotten T, Bakker M, van Hagen MP et al. Clinical manifestations of patients with intraocular inflammation and positive QuantiFERON-TB gold in-tube test in a country nonendemic for tuberculosis. Am J Ophthalmol 2014; 157(4): 754-761.

13 Triasih R, Robertson C, Duke T, Graham SM. Risk of infection and disease with Mycobacterium tuberculosis among children identified through prospective community-based contact screening in Indonesia. Trop Med Int Health 2015; 20(6): 737-743.

14 Harada N, Higuchi K, Yoshiyama T, Kawabe Y, Fujita A, Sasaki $Y$ et al. Comparison of the sensitivity and specificity of two whole blood interferon-gamma assays for $M$. tuberculosis infection. J Infect 2008; 56(5): 348-353.

15 Ganesh SK, Roopleen, Biswas J, Veena N. Role of highresolution computerized tomography (HRCT) of the chest in granulomatous uveitis: a tertiary uveitis clinic experience from india. Ocul Immunol Inflamm 2011; 19(1): 51-57.

16 Indonesian Society of Respirology. Diagnosis and Treatment Guidelines of Tuberculosis (TB) in Indonesia (TB consensus). Indonesian Society of Respirology: Jakarta, 2011.

17 The Directorate General of Disease Control and Environmental Health Ministry of Health Republic of Indonesia. National Guidelines of Tuberculosis Control. Ministry of Health Republic of Indonesia: Jakarta, 2014.

18 Ang M, Hedayatfar A, Zhang R, Chee SP. Clinical signs of uveitis associated with latent tuberculosis. Clin Exp Ophthalmol 2012; 40(7): 689-696.

19 Manousaridis K, Ong E, Stenton C, Gupta R, Browning AC, Pandit R. Clinical presentation, treatment, and outcomes in presumed intraocular tuberculosis: experience from Newcastle upon Tyne, UK. Eye 2013; 27(4): 480-486.

20 Manandhar A. Patterns of uveitis and scleritis in Nepal: a Tertiary Referral Center study. Ocul Immunol Inflamm 2016; e-pub ahead of print 18 May 2016; doi:10.3109/09273948. 2016.1161804.

21 Yeo TK, Ho SL, Lim WK, Teoh SC. Causes of visual loss associated with uveitis in a singapore tertiary eye center. Ocul Immunol Inflamm 2013; 21(4): 264-269.

22 Singh R, Gupta V, Gupta A. Pattern of uveitis in a referral eye clinic in north India. Indian J Ophthalmol 2004; 52(2): 121-125.

23 Win MZ, Win T, Myint S, Shwe T, Sandar H. Epidemiology of uveitis in a tertiary eye center in Myanmar. Ocul Immunol Inflamm 2016; e-pub ahead of print 11 March 2016; doi:10.3109/09273948.2015.1133839.

24 Al Dhahri H, Al Rubaie K, Hemachandran S, Mousa A, Gikandi PW, Al-Mezaine HS et al. Patterns of uveitis in a university-based Tertiary Referral Center in Riyadh, Saudi Arabia. Ocul Immunol Inflamm 2014; 1-9.

25 Jakob E, Reuland MS, Mackensen F, Harsch N, Fleckenstein $\mathrm{M}$, Lorenz HM et al. Uveitis subtypes in a german interdisciplinary uveitis center-analysis of 1916 patients. J Rheumatol 2009; 36(1): 127-136.

26 Rao NA, Saraswathy S, Smith RE. Tuberculous uveitis: distribution of Mycobacterium tuberculosis in the retinal 
pigment epithelium. Arch Ophthalmol 2006; 124(12): 1777-1779.

27 Wroblewski KJ, Hidayat AA, Neafie RC, Rao NA, Zapor M. Ocular tuberculosis: a clinicopathologic and molecular study. Ophthalmology 2011; 118(4): 772-777.

28 Bhuibhar SS, Biswas J. Nested PCR-positive tubercular ampiginous choroiditis: a case report. Ocul Immunol Inflamm 2012; 20(4): 303-305.

29 Balne PK, Modi RR, Choudhury N, Mohan N, Barik MR, Padhi TR et al. Factors influencing polymerase chain reaction outcomes in patients with clinically suspected ocular tuberculosis. J Ophthal Inflamm Infect 2014; 4(1): 10

30 Arora SK, Gupta V, Gupta A, Bambery P, Kapoor GS, Sehgal S. Diagnostic efficacy of polymerase chain reaction in granulomatous uveitis. Tuber Lung Dis 1999; 79(4): 229-233.

31 Bajgai P, Sharma K, Bansal R, Gupta N, Sharma A, Gupta A. Detection of Mycobacterium tuberculosis genome in subretinal fluid of patients with latent tuberculosis infection. Ocul Immunol Inflamm 2016; 24(6): 615-620.

32 Scheepers MA, Lecuona KA, Rogers G, Bunce C, Corcoran C, Michaelides $M$. The value of routine polymerase chain reaction analysis of intraocular fluid specimens in the diagnosis of infectious posterior uveitis. Sci World J 2013; 2013: 545149.

33 Geluk A, van Meijgaarden KE, Franken KL, Wieles B, Arend SM, Faber WR et al. Immunological crossreactivity of the Mycobacterium leprae CFP-10 with its homologue in Mycobacterium tuberculosis. Scand J Immunol 2004; 59(1): 66-70.
34 Geluk A, van Meijgaarden KE, Franken KL, Subronto YW, Wieles B, Arend SM et al. Identification and characterization of the ESAT-6 homologue of Mycobacterium leprae and T-cell cross-reactivity with Mycobacterium tuberculosis. Infect Immun 2002; 70(5): 2544-2548.

35 Rendini T, Levis W. Quantiferon-gold tuberculosis test cannot detect latent tuberculosis in patients with leprosy. Clin Infect Dis 2015; 61(9): 1439-1440.

36 Global leprosy update, 2015: time for action, accountability and inclusion. Wkly Epidemiol Rec 2015; 91(35): 405-420.

37 Gineys R, Bodaghi B, Carcelain G, Cassoux N, Boutin le TH, Amoura $Z$ et al. QuantiFERON-TB gold cut-off value: implications for the management of tuberculosis-related ocular inflammation. Am J Ophthalmol 2011; 152(3): 433-440. e431.

38 Jakob E, Max R, Zimmermann S, Dalpke AH, Alle W, Becker $\mathrm{M}$ et al. Three years of experience with QuantiFERON-TB gold testing in patients with uveitis. Ocul Immunol Inflamm 2014; 22(6): 478-484.

39 Agrawal R, Gonzalez-Lopez JJ, Nobre-Cardoso J, Gupta B, Grant R, Addison PK et al. Predictive factors for treatment failure in patients with presumed ocular tuberculosis in an area of low endemic prevalence. Br J Ophthalmol 2016; 100(3): 348-355.

40 Aabye MG, Ravn P, PrayGod G, Jeremiah K, Mugomela A, Jepsen $\mathrm{M}$ et al. The impact of HIV infection and CD4 cell count on the performance of an interferon gamma release assay in patients with pulmonary tuberculosis. PLOS ONE 2009; 4(1): e4220.

Supplementary Information accompanies this paper on Eye website (http://www.nature.com/eye) 